\title{
Perceptual grouping operates independently of attentional selection: Evidence from hemispatial neglect
}

\author{
SARAH ShOMSTeIN \\ George Washington University, Washington, D.C. \\ RuTH KIMCHI \\ University of Haifa, Haifa, Israel \\ MaXim Hammer \\ University of Pittsburgh Medical Center, Pittsburgh, Pennsylvania \\ and University of Pittsburgh, Pittsburgh, Pennsylvania \\ AND \\ Marlene BehrmanN \\ Carnegie Mellon University, Pittsburgh, Pennsylvania
}

\begin{abstract}
To what extent can human observers process visual information that is not currently the focus of attention? We evaluated the extent to which unattended visual information (i.e., that which appears on the neglected side of space in individuals with hemispatial neglect) is perceptually organized and influences the perceptual processing of information on the attended side. To examine this, patients (and matched controls) judged whether successive, complex checkerboard stimuli (targets), presented entirely to their intact side of space, were the same or different. Concurrent with this demanding task, irrelevant distractor elements appeared on the unattended side and either changed or retained their perceptual grouping on successive displays, independently of changes in the ipsilesional task-relevant target. Changes in the grouping of the unattended task-irrelevant distractor elements produced congruency effects on the attended target-change judgment to the same extent in the neglect patients as in the control participants, and this was true even in those patients with severe attentional deficits. These results suggest that some perceptual processes, such as grouping, can operate in the absence of attention.
\end{abstract}

Representing the visual world efficiently requires selecting a fraction of the multitude of information that is available to the visual system at any one instant in time. Much recent research has been devoted to understanding the psychological and neural processes underlying the attentional selection of incoming information (Corbetta \& Shulman, 2002; Reynolds \& Heeger, 2009; Shomstein \& Yantis, 2006; Yantis et al., 2002), but there still remains considerable controversy concerning the fate of the sensory information that is not selected for preferential processing. That is, when an observer selectively attends to some subset of the visual input, the degree and nature of the processing to which the remaining, unattended input is subjected remains largely unspecified. Part of the problem in deriving a definitive answer to this issue arises first from the difficulty in knowing with certainty that the unselected information is, in fact, unattended, and second from developing a paradigm that targets just this unattended information.

It is not particularly contentious that, after the light hits the retina, some amount of visual processing is accomplished, regardless of whether the specific information is attended or not. What remains more uncertain, however, is the extent to which the unattended information is processed perceptually (e.g., segmented into figure/ground, grouped, etc.). Previous investigations of attentional involvement in different types of perceptual processing vary from studies of how scenes are perceived (Biederman, Rabinowitz, Glass, \& Stacy, 1974; Grill-Spector \& Kanwisher, 2005; Potter \& Fox, 2009; Torralba, 2003), how memories of pictures/scenes are constructed (Wolfe, Horowitz, \& Michod, 2007), and how visual categorization occurs (Li, VanRullen, Koch, \& Perona, 2002; Walker, Stafford, \& Davis, 2008). Despite the number of studies 
that have explored this issue, there is not a clear consensus on the outcome. For example, some studies have demonstrated that whether or not a natural scene contains an animal can be determined under very brief exposure duration and is "attention free" (e.g., Li et al., 2002), whereas others claim that these types of perceptual processes do require attention (e.g., Walker et al., 2008).

Just as the debate about the role of attention in object and/or semantic processing is ongoing, there is a similar ongoing debate about the role of attention in even more foundational perceptual processing: perceptual organization. A strong claim made by some researchers is that little, if any, visual processing occurs in the absence of attention and that perception cannot proceed without attentional selection (Mack \& Rock, 1998; Mack, Tang, Tuma, Kahn, \& Rock, 1992). Evidence supporting this account is drawn from inattention paradigms (Mack \& Rock, 1998) in which task-irrelevant grouped items went unnoticed and did not influence the behavioral performance of the observers. In one well-known version of this paradigm, Mack and Rock had observers judge whether the horizontal or vertical arm of a briefly flashed cross was longer. On the fourth trial, an unexpected texture gradient (grouped by similarity, proximity, or motion) was presented simultaneously with the cross but at a different position on the screen. When the cross was presented at fixation and the texture gradient was presented parafoveally, about one quarter of the observers failed to perceive the presence of the unexpected object. Even more startling, when the cross was presented parafoveally and the texture gradient was presented at fixation, nearly three quarters of the observers failed to detect the unexpected object. These findings suggest that, in the absence of attention, information is not processed and, therefore, perception fails (see also Enns \& Kingstone, 1995).

An equally strong but opposing account suggests that fundamental visual processes, such as perceptual grouping and figure-ground segmentation, can, in fact, take place in the absence of attention (Driver, Davis, Russell, Turatto, \& Freeman, 2001; Kimchi \& Peterson, 2008; Kimchi \& Razpurker-Apfeld, 2004; Lamy, Segal, \& Ruderman, 2006; Moore \& Egeth, 1997; Russell \& Driver, 2005). In one illustrative study, participants judged which of two parallel lines superimposed on a background matrix of black and white dots, was longer (Moore \& Egeth, 1997). The background dots whose presence was orthogonal to the linejudgment task were either randomly colored or grouped to form the Müller-Lyer illusion. Because the length judgment was clearly influenced by whether the background dots gave rise to the illusion, even though the participants were not aware of the illusion, the authors concluded that perceptual grouping could occur without attention. Further support for the view that perceptual grouping occurs without attention comes from recent studies in which participants performed a change detection task on a small, centrally located, black-and-white matrix, presented for a brief duration (Kimchi \& Razpurker-Apfeld, 2004; Russell \& Driver, 2005). The matrix-like target stimulus was embedded in the center of a background pattern consisting of colored dots that were grouped by similarity into rows, columns, or simple shapes. On each trial, two suc- cessive displays were presented, and participants judged whether the central target matrix remained the same or changed. Additionally, within a trial, the organization of the background elements stayed the same or changed independently of the status of the target matrix. Of greatest relevance was the finding that the grouping of the background stimuli (whether it stayed the same or changed across successive displays) influenced the detection of changes in the target matrix - even though, when probed with surprise questions, participants could report neither the background grouping nor its change-leading to the conclusion that unattended background elements were perceptually grouped (see also Lamy et al., 2006).

A concern in any attempt to examine processing without attention is whether information is truly "unattended." 1 In the studies listed above, the definition of unattended typically relied on the following criteria: The target task was demanding enough to absorb sufficient attention, the background grouping was entirely irrelevant to the target task, and, importantly, participants self-reported that the task-irrelevant information was not perceived, indicating inattentional blindness (Mack \& Rock, 1998). Self-report information is often useful in understanding the nature of perception, but it is notoriously problematic (Merikle, Smilek, \& Eastwood, 2001), so it remains difficult to ensure and verify that the information is truly unattended. In this study, the definition of unattended is independent of the experimental manipulations, and we circumvented the shortcomings of self-report by examining the perception of information on the left side of space in individuals who, due to a cortical lesion, were profoundly impaired at attending to the left side of space. This disorder, termed hemispatial neglect (neglect), reflects the failure to attend to information appearing on the contralesional left side (for recent reviews, see Hillis, 2006; Parton, Malhotra, \& Husain, 2004), and we characterize the degree to which the individuals in our study failed to detect information on the contralesional side prior to their participation in the experiments of interest reported here (see Figure 1). Investigating the behavior of individuals with hemispatial neglect provides a unique opportunity to address the issues at hand - namely, the extent to which the neglected or unattended information is perceptually organized.

To investigate whether unattended information undergoes perceptual grouping, we modified the paradigm that was originally described in Driver et al. (2001; see full details in Russell \& Driver, 2005) and adopted by Kimchi and Razpurker-Apfeld (2004). On each trial, observers were presented with two brief successive target displays that were positioned to the right of the fixation point and consisted of a checkerboard-like pattern made up of random black and white squares. The observer's task was to judge whether the two successive target displays were the same or different. Note that the checkerboard squares were placed entirely in the patients' intact right hemispace, obviating the need to process any contralesional information. It is also important to note that this task was highly demanding since, in the different trials, the target displays differed only by a displacement of one of the internal black or white squares. Concurrent with the target display, 

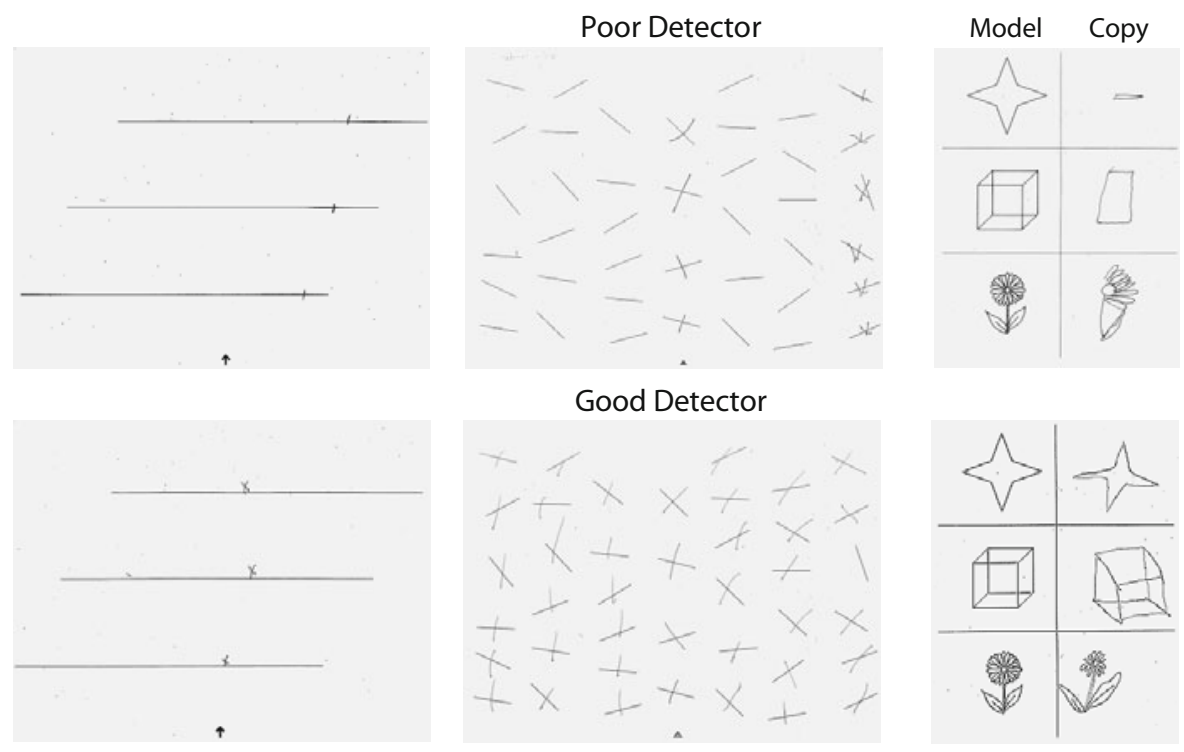

Figure 1. Two patients' performance on line bisection (left panel), line cancellation (middle panel), and copying (right panel) tests taken from the Behavioural Inattention Test. For the line cancellation task, the middle row of lines was canceled by the experimenter as a means of instructing the patient on the procedure. The labels "good detector" and "poor detector" indicate the classification of the patient following their performance on the attentional-cuing task.

participants were presented with a task-irrelevant stimulus that was positioned to the left of the fixation point and consisted of grayscaled dots grouped by common color into rows/columns or squares/crosses. The organization of the distractor elements stayed the same or changed across successive displays, independently of whether successive targets were the same or different.

If grouping occurs without attention, then the behavior of neglect patients on ipsilesional target-change detection could nonetheless be influenced by the contralesional items, despite those patients' left-sided attentional impairment. This ipsilesional-contralesional response compatibility should manifest as follows: Target-different responses should be faster when the grouping of the distractors differs on successive presentations (different ipsilesional and different contralesional) than when it stays the same (different ipsilesional and same contralesional), and target-same responses should be faster when the distractors' grouping stays the same (same ipsilesional and same contralesional) than when it changes (same ipsilesional and different contralesional).

Here, we report new evidence that is consistent with this congruency prediction: The data obtained from the patients reveal that unattended stimuli presented to the neglected contralesional side nonetheless undergo perceptual organization, as indicated by the influence of changes in the organization of unattended information on the targetchange detection of individuals with neglect. Note that, in individuals whose neglect is so severe that their ability to detect the presence of contralesional information is severely impaired, unattended stimuli affected performance to the same degree as in individuals with mild neglect and in normal matched control participants.

\section{METHOD}

\section{Attentional Cuing Paradigm Task}

Participants. Eight patients with chronic, right-lateralized, focal cortical lesions and 10 healthy control participants (matched to the patients on age and education levels) consented to participate in the experiments, in accordance with the protocol approved by the Institutional Review Boards of Carnegie Mellon University and the University of Pittsburgh. All patients had normal or corrected-to-normal vision, were tested at least 8 months following the onset of the cerebrovascular incident, and ranged between 42 and 78 years of age. All patients scored below 100 (cutoff 132/146 for neglect) on the Behavioural Inattention Test (BIT; Wilson, Cockburn, \& Halligan, 1987), meeting the criterion for hemispatial neglect. The BIT includes line cancellation, letter cancellation, star cancellation, figure and shape copying, line bisection, and representational drawing tasks, thereby sampling neglect across a wide array of visuoperceptual tasks (see Figure 1). Further description of the patients' attentional profiles is provided in the Results section.

Apparatus and Stimuli. To confirm the presence of an attentional disorder and to quantify objectively the severity of the deficit, the attentional behavior of each individual was characterized using a well-established covert attentional-cuing paradigm (Friedrich, Egly, Rafal, \& Beck, 1998; Posner, Snyder, \& Davidson, 1980; Posner, Walker, Friedrich, \& Rafal, 1984). Stimuli were displayed on a 19-in. color monitor situated roughly $62 \mathrm{~cm}$ from the observer. Displays consisted of a central fixation point and two outline boxes positioned approximately $2.5^{\circ}$ to the right and left of fixation. Each box subtended $1.8^{\circ}$ and was drawn in light gray ink on a black background. The cue consisted of a brief brightening of one of the boxes (i.e., outline of the box changing from gray to white). The target, which was presented within one of the boxes, was a small asterisk subtending $0.5^{\circ}$. The experiment consisted of four conditions: valid, in which the target appeared at the same location as the cue; invalid, in which the target appeared at the location opposite the cue; neutral, in which both boxes were cued and the target was presented randomly in one of the boxes; and catch trials, in which one or two boxes were cued, but no target was ever presented ( $10 \%$ of the total number of trials). There were 
equal numbers of valid, neutral, and invalid trials. When only one cue appeared, valid and invalid trials were equally likely, and the side of the target and the cue were equiprobable across all trials. Visual feedback was provided on every trial as follows: "correct" for hits, "incorrect" for false alarms (or catch trials), and "no response detected" for misses (patients were confirmed not to have neglect dyslexia).

Procedure. The two boxes were always displayed on the screen, and the beginning of the trial was signaled by the onset of a central fixation point for $500 \mathrm{msec}$, followed by a $200-\mathrm{msec}$ cue. On targetpresent trials, after a variable cue-to-target interval ( 50 or $150 \mathrm{msec}$ ), the target was presented in one of the boxes and remained visible until response or 3,000 msec had elapsed. Each trial was followed by a feedback screen for $2 \mathrm{sec}$ and was separated from the subsequent trial by a 2 -sec interval.

Participants were told that the cue was uninformative (i.e., that it would predict the location of the target with $50 \%$ accuracy) and were required to maintain fixation and respond quickly without sacrificing accuracy. Responses were made with the right hand by pressing the space bar on the keyboard. Testing sessions consisted of 10 practice trials followed by 12 blocks of 84 trials each.

\section{Sequential Matching Task}

Participants. The participants in the attentional cuing paradigm task also took part in the sequential matching task.

Apparatus and Stimuli. Observers performed a same/different sequential matching task on two briefly presented successive checkerboard displays (targets) presented ipsilesionally $0.7^{\circ}$ (fixation to left edge) to the right of the fixation point (see Figures 2A and 2B). The checkerboard target consisted of 16 black and 9 white, small, solid squares, $0.37^{\circ}$ each, randomly located in a $5 \times 5$ matrix subtending $2^{\circ} \times 2^{\circ}$. On half of the trials, the target was the same on the successive displays. On the remaining half of the trials, the targets differed across the successive displays, with one of the white squares having been moved into an adjacent position, ${ }^{2}$ chosen randomly and equiprobably on the left and right of the checkerboard.

Simultaneously with the checkerboard, a task-irrelevant distractor appeared $0.7^{\circ}$ to the left of the fixation point in the cross-hemifield condition (Figures 2A and 2B) or above or below the checkerboard in the same-hemifield condition (Figure 2D). The same-hemifield condition provided an indication of the ipsilesional strength of the distractor influence on the target and enabled us to compare it with the contralesional strength of the distractor influence from the crosshemifield condition. This task-irrelevant distractor fell into one of two conditions. In the rows/columns $\times$ color similarity condition (Figure 2C), the distractor consisted of an array of black and light gray dots organized into rows or columns in a $6 \times 6$ matrix subtending $2^{\circ} \times 2^{\circ}$. The color of the dots always changed across the two successive displays of a trial (i.e., black dots turned into white, and the light gray dots turned into dark gray) so that a change of organization would not be confounded with a change in the color of the dots. On half of the trials, the perceptual organization of the dots remained the same (i.e., rows to rows, columns to columns), whereas, on the remaining half, the organization changed (i.e., rows into columns and vice versa). Note that, because the color of the dots changed on every trial, no local information was ever informative about the grouping status of the display; it is only by virtue of the global organization into rows or columns that the organization of the unattended elements stayed the same or changed across successive displays. In the cross/square $\times$ color similarity condition (Figure 2C), the distractor consisted of either a cross or a square shape, made of black or white dots. As in the rows/columns condition, the color of the dots changed from the first to second display. Additionally, the cross/square changed in shape on half of the trials. Changes in the distractors' grouping occurred independently of any change in the target, making the grouping of the distractor elements entirely irrelevant to the task performed by the observer. The use of two different forms of perceptual organization-rows/columns versus crosses/squares - provided a measure of the generality and variety of perceptual grouping that might potentially have occurred in the absence of attention.

Design and Procedure. Participants completed the samehemifield and cross-hemifield conditions in separate sessions with 8 blocks of 40 trials in each condition. Distractor organization (rows/ columns, crosses/squares) was also blocked. All combinations of
A

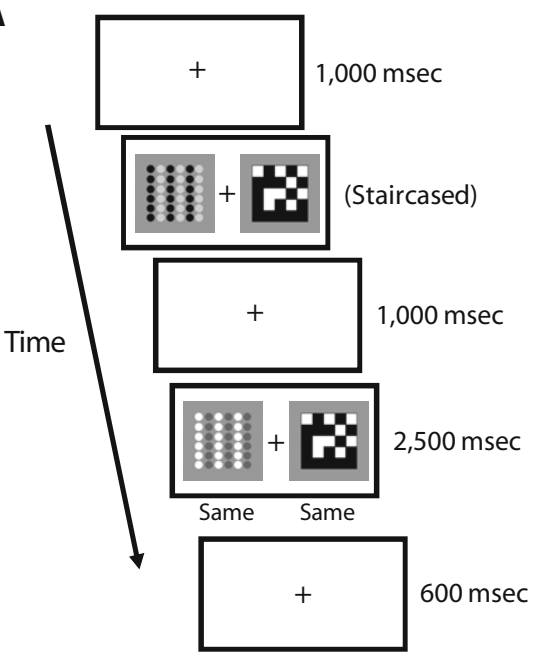

B

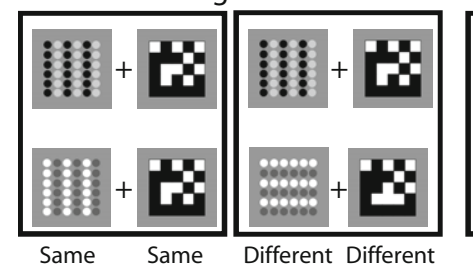

Incongruent

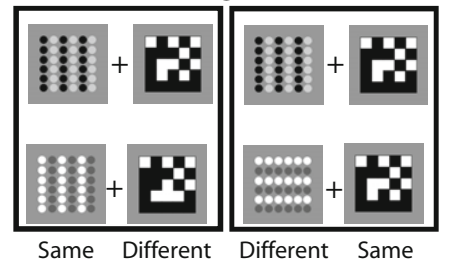

C

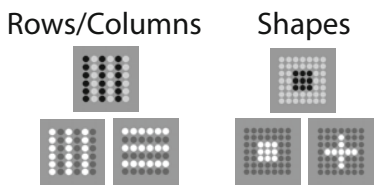

D Same-Hemifield

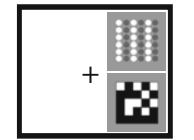

Figure 2. Example displays for the checkerboard sequential-change detection paradigm. (A) Sequence and timing of an experimental trial. The illustration depicts a congruent same-target same-distractor trial. (B) Examples of different permutations of target and distractor congruencies in successive displays. (C) Different types of distractor organizations. (D) Example of a same-hemifield target-distractor condition. Note that the color of the distractor dots always changes across the two successive displays of a trial (i.e., black dots turned into white, and the light gray dots turned into dark gray), so that a change in organization is not confounded with a change in the color of the dots. 
distractor and target congruency were randomized within blocks, with each combination occurring on an equal number of trials.

Each trial began with a 1-sec fixation point presented centrally, followed by the first display (a checkerboard and a task-irrelevant distractor), which remained on the screen for a variable duration. This display duration was titrated (via a staircasing procedure for each condition) to yield an overall $85 \%$ target accuracy, and, thus, response time (RT), rather than accuracy, was the measure of interest. ${ }^{3}$ Staircasing was employed to ensure that participants allocated maximal attentional resources to the task-relevant checkerboard stimulus. After a 1-sec delay, the second display (a checkerboard and a task-irrelevant distractor) was presented for $2,500 \mathrm{msec}$. Responses were collected from target onset (left mouse click for same responses and right mouse click for different responses) and were permitted for up to $6,000 \mathrm{msec}$ after target offset, after which time the next trial was initiated.

\section{RESULTS}

\section{Characterizing the Attentional Deficit}

In order to confirm the presence of an attentional disorder (rather than a sensory deficit) beyond that of the bedside screening measure, and to objectively quantify the severity of the deficit, the attentional behavior of each individual was characterized using a well-established covert attentional-cuing paradigm (Friedrich et al., 1998; Posner et al., 1980; Posner et al., 1984) (see the Method section for details). Preliminary analysis indicated no significant main effect of SOA (on accuracy or RT) and no interaction of SOA with any other factor $(F<1)$; therefore, the data were collapsed over SOA. On the basis of the accuracy of detecting targets appearing on the left side of space, as determined by this attentional-cuing paradigm, patients were divided into two groups (4 patients in each; see Figures 3 and 4): the good detectors (GD), who exhibited high accuracy, regardless of the side of space on which the target appeared $(F<1)$, and the poor detectors (PD), who exhibited significantly lower accuracy (responding to roughly half of the targets) on the left than on the right side $[F(1,3)=12.12, p<.04]$. Consistent with this split, which was based on the severity of the attentional disorder, PD patients exhibited lower BIT scores than GD patients did on all items administered: line crossing $(M=7$ vs. $M=11)$, letter cancellation $(M=3$ vs. $M=9)$, star cancellation $(M=8$ vs. $M=$ $17)$, and line bisection $(M=1.5$ vs. $M=2)$.

The data from this covert attentional-cuing paradigm were subjected to a repeated measures ANOVA with median RT as the dependent measure, target side (left or right) and validity (valid, neutral, invalid) as within-subjects factors, and group (PD, GD, controls) as a between-subjects factor. Note that RTs on trials with stimuli presented to the neglected side of the PD group are not obviously interpretable, since the RTs most likely stemmed from an arbitrary chance $(50 / 50)$ decision of whether to press the response button on any given trial when the target was presented in the neglected hemifield. Nevertheless, ANOVAs revealed significant two-way interactions of target side $X$ group $[F(2,15)=28.07, p<.001]$ and target side $\times$ validity $[F(2,30)=9.76, p<.001]$, as well as a three-way interaction of target side $\times$ validity $\times$ group $[F(4,30)=$ $6.51, p<.001]$. The control group replicated the previously established pattern of performance (Posner et al., 1980), with a significant RT cost for the invalid over valid trials, but with no left-right difference (Figure 3). Relative to the controls', reactions of patients from both the PD and GD groups were disproportionately slowed when targets appeared on the contralesional left side (i.e., neglected), as compared with when the targets appeared on the ipsilesional right side of space (Friedrich et al., 1998; Posner et al., 1984). This left-right asymmetry is especially evident in the case of neutral and invalid trials, and this confirms the diagnosis of left-sided neglect, manifest to differing degrees between each patient group and controls, as well as to differing degrees between the GD and PD groups. ${ }^{4}$

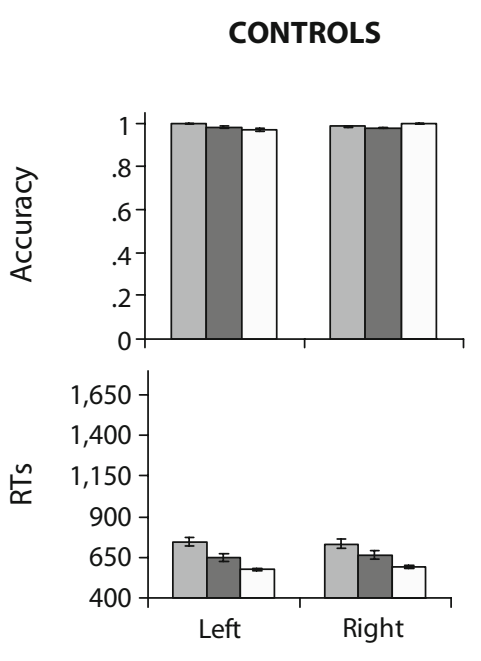

Target Location

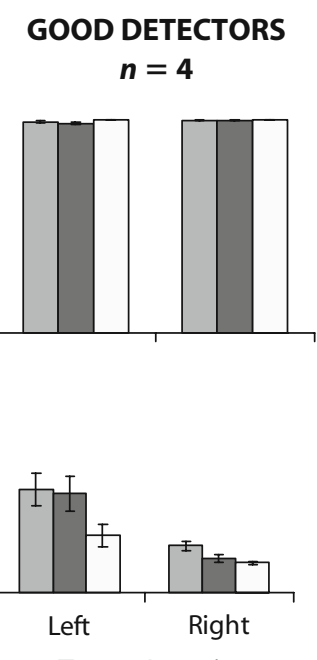

Target Location
POOR DETECTORS $n=4$
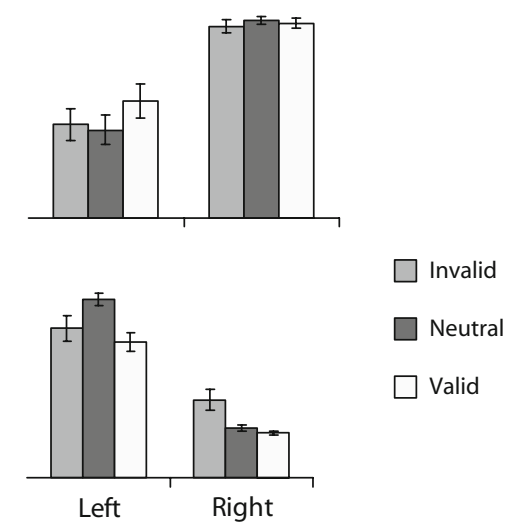

Target Location

Figure 3. Accuracy (top panel) and reaction times (RTs, in milliseconds; bottom panel) for controls, good detectors, and poor detectors in the Posner cuing task. Error bars represent standard errors of the means. 


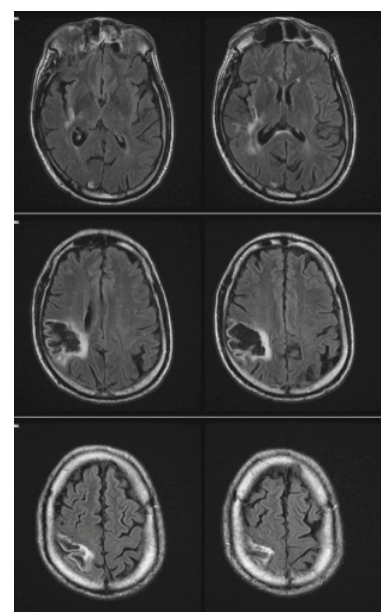

Patient 1

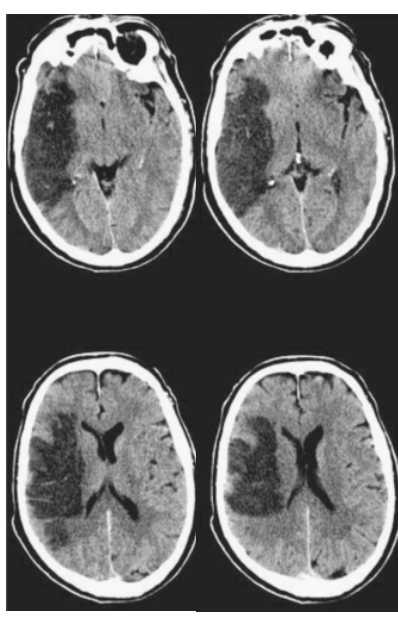

Patient 2

Poor Detectors

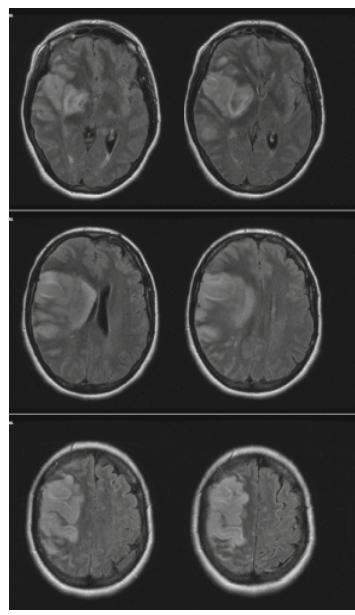

Patient 1

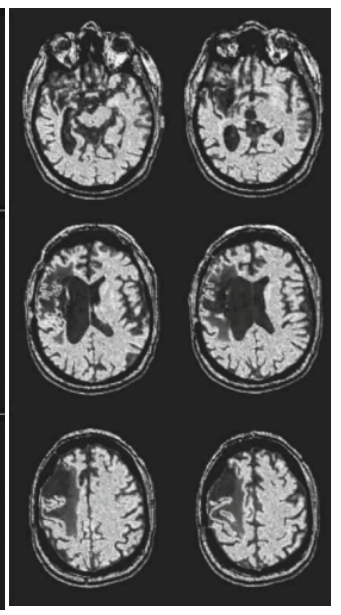

Patient 2

Good Detectors

Figure 4. A sample of representative anatomical scans (T2 flair and CT scans) for 2 patients from the poor detector group (left panel) and 2 patients from the good detector group (right panel). Scans are presented in the radiological convention (i.e., left is right). All patients had unilateral right-side lesions.

\section{Perceptual Processing on the Neglected Side}

Having classified the performance of individuals on the basis of severity, we now examine the extent to which stimuli presented to the unattended or neglected side were processed and whether this differed across the GD and PD groups. Specifically, by assessing the extent to which contralesional task-irrelevant distractors influenced change detection of targets presented on the intact ipsilesional side, we examined whether perceptual grouping could be achieved in the absence of attention. If neglected stimuli can be perceptually organized, we should observe an influence of the irrelevant distractor on target-change judgment on the intact right side in both patient groups. That is, we should see the following congruence effects: When the distractor retains its organization across successive displays, it should facilitate responses in target-same trials and slow target-different trials, whereas, when the distractor changes its organization across successive displays, the converse should be true. Furthermore, if perceptual grouping can occur in the absence of attention, the magnitude of distractor congruence effects should be equivalent across all groups (GD, PD, controls), independent of the severity of the attentional deficit.

The median RT data for correct responses (correct responses to ipsilesional targets) were subjected to an omnibus ANOVA. Preliminary ANOVAs revealed no differences between the PD and GD groups, nor between the rows/columns and shapes (neither main effects nor interactions with these factors), and, therefore, the following analysis was conducted collapsing over these factors.

The ANOVA was conducted with hemifield of distractor (same-hemifield, cross-hemifield), target (same, different within a trial), and distractor (same, different within a trial) as within-subjects factors, and group (patients, controls) as a between-subjects factor (see Figure 5). A significant main effect of group $[F(1,16)=46.63, p<.001]$ was observed, with patients exhibiting significantly slower aver- age RTs ( $M=2,018 \mathrm{msec})$ than controls $(M=966 \mathrm{msec})$. Additionally, a significant target $\times$ distractor interaction was observed $[F(1,16)=67.27, p<.001]$, revealing that the congruency effects produced by the task-irrelevant distractor were larger for the target-different condition (targetdifferent responses were $117 \mathrm{msec}$ faster when the distractor's organization changed than when it stayed the same) as compared with those for the target-same condition (targetsame responses were $48 \mathrm{msec}$ faster when the distractor's organization stayed the same than when it changed). Furthermore, a significant group $\times$ target $\times$ distractor interaction was observed $[F(1,16)=32.07, p<.001]$, revealing that neglect patients exhibited greater congruency effects from the task-irrelevant distractor in the target-different condition (target-different responses were $173 \mathrm{msec}$ faster when the distactor's organization changed than when it stayed the same for the patients, as compared with $61 \mathrm{msec}$ for controls), and in the target-same condition, congruency effects were observed only for the patients (target-same responses were $121 \mathrm{msec}$ faster when the distractor's organization stayed the same than when it changed for the patients, as compared with $-24 \mathrm{msec}$ for controls). In addition, we observed a marginally significant hemifield $X$ target $\times$ distractor $\times$ group interaction $[F(1,16)=4.3, p=$ $.054]$; as compared with controls, patients exhibited greater congruency effects from an irrelevant distractor when distractors were positioned in the neglect (i.e., left) hemifield. In summary, the pattern of results observed for patients and controls were similar; task-irrelevant distractors produced congruency effects for controls, whose attention was evenly distributed across the two hemifields, as well as for patients, whose attention was heavily biased toward the right hemifield (where the targets were positioned).

Due to the large differences in base RTs exhibited by the patient and control groups (i.e., main effect of group), however, the finding of differing magnitude of influence of distractor on the target change detection for the two 


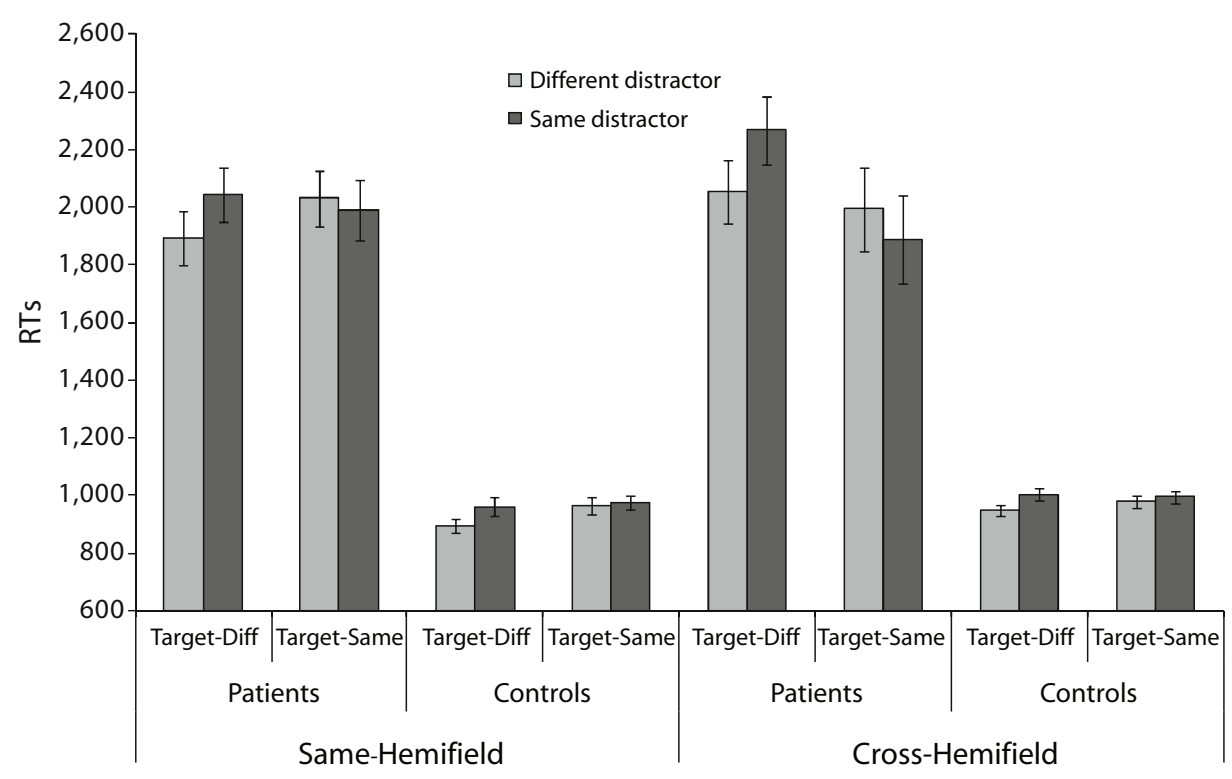

Figure 5. Raw median correct reaction times (RTs, in milliseconds) for same and different targets for same-hemifield and cross-hemifield distractor conditions in the sequential matching task. Error bars represent standard errors of the means.

groups revealed that using absolute RTs as the dependent measure may potentially be misleading. That is, one has to be cautious when interpreting greater distractor influence in patients than in controls because the effects might simply be scaled by the magnitude of the overall RTs. To more directly examine whether the magnitude of the distractor influence differs across groups, and to take into account these large RT differences among groups (Figure 5), we calculated a normalized congruence score for each participant and used this score as the dependent measure in the subsequent ANOVAs.

To this end, we derived a normalized congruence score for each individual for each target condition by subtracting target-distractor congruent condition RTs (i.e., same target-same distractor and different target-different distractor) from the target-distractor incongruent condition RTs (i.e., same target-different distractor and different target-same distractor). The congruence score was then divided by the mean of the RTs in that condition and multiplied by 100 , thus factoring out the effect of varying base RTs for each individual (see Figure 6). ${ }^{5}$

The normalized congruence score for each participant was used as the dependent measure in an omnibus ANOVA with hemifield (same-hemifield, cross-hemifield) and target (same, different) as within-subjects factors and group (PD, GD, controls) as a between-subjects factor (see Figure 6). We included three groups in this analysis, rather than collapsing over the two patient groups (PD, GD) on the basis of the raw RT findings, in order to investigate whether distractor influence, as measured by the scaled congruence score, might differ across the two patient groups. Data were collapsed over distractor organization (rows/columns, cross/square) because preliminary analyses did not show a main effect or any interactions with this factor $\left(F_{\mathbf{S}}<1\right)$. A significant main effect of target $[F(1,15)=18.27, p<.01]$, with larger congruence scores for target-different than for target-same trials, was observed. There was also a significant main effect of group, with larger congruence scores for both patient groups than for controls $[F(2,15)=5.89$, $p<.02]$ (mean congruence scores of 5.8 and 7.0 for PD and GD groups, respectively, compared with a score of 2.1 for the control group), but no difference between the patient groups themselves $(F<1)$.

Planned comparisons were conducted in order to examine differences between groups and conditions for each hemifield. Of great interest, no significant differences between any groups or between conditions were observed in the same-hemifield condition (i.e., neither a significant main effect of group, nor an interaction of group with any other factor). In contrast, we observed significant differences between groups for the distractors presented in the cross-hemifield condition. For the target-different condition, a marginally significant difference between patients (PD and GD combined) and controls $[t(12)=1.85$, $p=.08]$ was observed, with congruence scores greater for the former $(M=10.59)$ than for the latter $(M=5.48)$. In addition, congruence scores differed with marginal significance between GDs and controls $[t(12)=2.08, p<.06]$ and between PDs and controls $[t(12)=1.02, p=.05]$, such that both patient groups were more susceptible to distractor influence than controls were. For the target-same condition, patients as a group exhibited a greater congruency effect $(M=5.6)$ than did controls $(M=-1.7)[t(16)=2.75$, $p<.02]$. In addition, a significant difference between PDs and controls was observed $[t(12)=2.36, p<.05]$, with congruence scores of $M=6.46$ for PDs and $M=-1.7$ for controls. GDs also showed a statistically marginal difference from controls $[t(12)=1.8, p<.09]$.

These results indicate that the magnitude of distractor influence does not vary as a function of neglect se- 

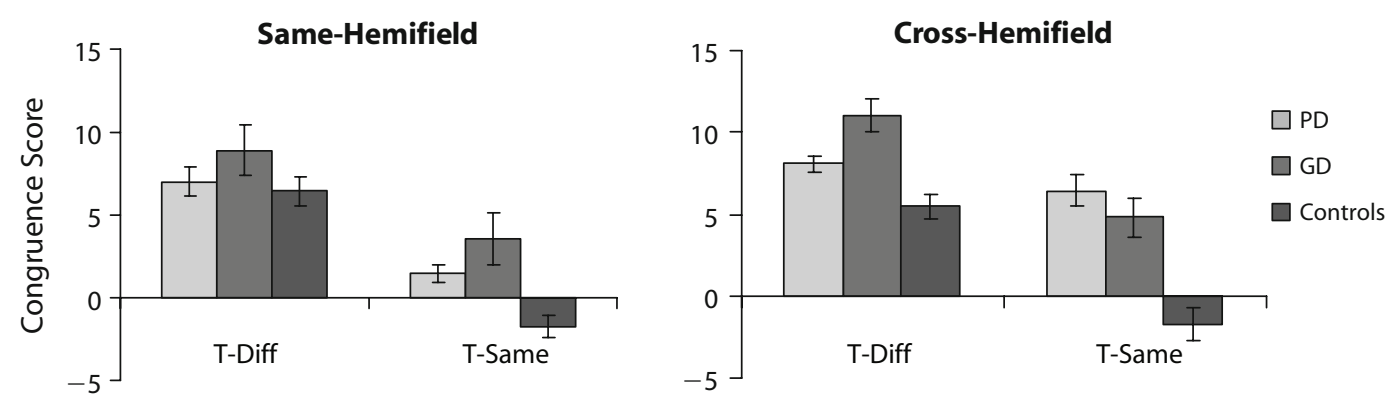

Figure 6. Derived congruence scores for same and different targets for same-hemifield and cross-hemifield distractors for controls, poor detectors (PD), and good detectors (GD). Error bars represent standard errors of the means.

verity (i.e., PDs did not show less influence of the taskirrelevant distractor than GDs did). Also, surprisingly, the patients did not show less influence of the task-irrelevant, neglected distractor than did the controls; instead, they tended to show a greater congruency effect as a group (patients were affected to a greater degree by task-irrelevant distractors than controls were). Patients with impaired attentional selection showed substantial processing of unattended stimuli, as reflected in the sensitivity of their performance to the task-irrelevant, unattended information. Moreover, and provocatively, the activation of contralesional distractors may be abnormally enhanced. As we discuss below, this suggests that, in neglect, in cases where contralesional information is not well represented, the unattended distractors may not be filtered out efficiently, and, consequently, contralesional stimuli may have greater influence than ipsilesional stimuli do in patients, relative to controls.

\section{DISCUSSION}

A longstanding issue that has generated vigorous debate among scholars of visual science is whether information that is not selected for preferential processing - and is, hence, unattended - is nevertheless processed and represented. The resolution to this issue has deep implications for understanding the mechanism by which external sensory information gains access to the perceptual system and, ultimately, influences behavior. The findings from this study clearly suggest that sensory information is perceptually organized, even in the absence of attention. Using a unique window into attentional function, provided by individuals who suffer from hemispatial neglect (a profound attentional impairment), we demonstrated that taskirrelevant, unattended distractors influenced task-relevant target processing in these individuals. Evidence to support this statement comes from the fact that changes in the distractors' organization (i.e., whether distractors retained or changed their perceptual grouping across successive displays) produced congruency effects on target-change judgments. Furthermore, and surprisingly, the congruency effects arising from changes in the distractors' grouping were roughly equivalent across the two groups of patients, independent of the severity of the attentional disorder. These findings lead to the conclusion that gestalt percep- tual grouping processes take place in the absence of attention. Two important observations follow from these results: (1) Neglected visual stimuli are indeed processed and can influence behavior, and (2) attentional selection plays a role not only in target selection but also in filtering out or suppressing distractors. Each of these conclusions has theoretical implications, and we consider each in turn.

\section{Perceptual Organization Occurs in the Absence of Attention}

The conclusion that perceptual grouping can take place in the absence of attentional selection is supported by the finding that a change in task-irrelevant distractor organization (e.g., columns to rows and vice versa, cross to square and vice versa) influenced target-change detection. This finding is especially dramatic, given that the distractors appeared contralesionally and that they were entirely task irrelevant. It is important to note that distractor influence cannot be explained by a simple local change of the distractor elements; distractor dots always changed their color across the successive displays. Thus, the preservation or alteration of the gestalt perceptual grouping of the colored dots in the task-irrelevant distractor served as the source of the distractors' influence, and this held for distractors where the dots were grouped by color similarity into either oriented stripes (i.e., columns and rows) or more complex shapes (i.e., cross and square).

A critical aspect of our claim is that the influence of the task-irrelevant distractors occurs in the absence of attention to this contralesional information. The extent to which the individuals with neglect- or even controlsare not attending to the neglected stimuli has been a source of much controversy in the literature. We have argued that the findings from the covert attentional-cuing task have confirmed the presence of an attentional deficit and the failure to process the contralesional stimuli in our participants. Given the deficit (which is severe in the PD patients), assessed independently of the main experiment, we have not had to rely on participants' self-report of awareness (or rather lack of awareness) of the distractors' organization, as has been true in many other investigations (e.g., Kimchi \& Razpurker-Apfeld, 2004; Moore \& Egeth, 1997; Russell \& Driver, 2005).

Note that the covert attentional-cuing task is direct in the sense that it relies on explicit detection of and responses 
to stimuli. Note, however, that the task in which we assessed the influence of the unattended distractors per se did not require self-report and, hence, may be considered an indirect task. So, while we use a direct task to classify and differentiate between the severe and more mild forms of neglect, in the main experimental paradigm, observers were not required to report any aspect of the unattended information, and performance was not "contaminated" by self-report.

Even though we have argued that there was no need whatsoever to attend to the contralesional distractors, it might still have been the case that the patients attended there overtly or covertly, even intermittently. If so, this would undermine our claim that the influence of the taskirrelevant distractors did not emerge from their having been processed in the absence of attention. However, the likelihood that the neglect patients overtly moved their eyes contralesionally is low. Many previous studies have documented an ipsilesional bias on saccades in these patients, with an abnormally larger number of fixations within the unaffected field than in the neglected fieldespecially in more severe cases of neglect, like those we included in the PD group (Behrmann, Barton, Watt, \& Black, 1997; Gainotti, De Luca, Figliozzi, \& Doricchi, 2009; Karnath \& Fetter, 1995), consistent with the notion of ipsilesional "hyperattention" or capture (Làdavas, Carletti, \& Gori, 1994; Làdavas, Petronio, \& Umiltà, 1990; Làdavas, Umiltà, Ziani, Brogi, \& Minarini, 1993). Indeed, in more severe patients, few, if any, exploratory eye movements are made to the neglected field - especially when a competing stimulus is presented on the ipsilesional right side - and so the probability of contralesional eye movements was particularly low in the patients in this study with severe attentional deficits. We also note, that if it were the case that the patients did move their eyes out of the ipsilesional field, this would give rise to many errors on the task-relevant checkerboard paradigm, which is not consistent with our data (all of the patients were able to achieve $85 \%$ accuracy).

There are also reasons to be skeptical about the possibility that the patients covertly directed their attention to stimuli other than the task-relevant checkerboard. There is considerable evidence that distractors are excluded from perception when the perceptual load of processing taskrelevant stimuli is sufficiently high to exhaust perceptual capacity, leaving little, if any, ability for distractor processing (Lavie, 1995; Lavie \& Robertson, 2001; Lavie \& Tsal, 1994). In addition to the support for this notion of perceptual load obtained from behavioral studies, further support is gleaned from Rees, Frith, and Lavie's (1997) study, which examined neural activity in visual cortices under high versus low perceptual load. In their study, using functional MRI, neural activity associated with the perception of irrelevant motion distractors (e.g., area MT/ V5) was reduced under higher load task of linguistic judgments performed on words presented at fixation (Rees et al., 1997). A similar finding was obtained in another imaging study, in which participants performed a foveal task of low or high attentional load. Irrelevant, invisible, monocular stimuli were simultaneously presented periph- erally and were continuously suppressed by a flashing mask in the other eye. Attentional load in the foveal task strongly modulated retinotopic activity evoked in primary visual cortex (V1) by the invisible stimuli, such that the activation in early visual cortex was reduced when the attentional load was high (Bahrami, Lavie, \& Rees, 2007).

The effect of load on awareness and conscious perception has also been evaluated in the context of the "inattentional blindness" paradigm, in which awareness of a taskirrelevant stimulus was significantly reduced by higher perceptual load (with increased numbers of search items, or a harder discrimination vs. detection task) (CartwrightFinch \& Lavie, 2007). Taken together, the findings from the different studies demonstrate that conscious perception of task-irrelevant stimuli critically depends on the level of task-relevant perceptual load. The paradigm that we have adopted corresponds to a high-perceptual-load task, as evidenced by target-performance accuracy fixed at $85 \%$ via the staircasing method. Our paradigm, therefore, has the added benefit that the attention to the task-irrelevant distractors is greatly reduced, even under the best of circumstances with normal observers, and probably even to a greater degree in patients who, left to their own devices, neglect the contralesional side anyway.

We also note that variants of the paradigm we have used here as an indirect method for assessing inattentive visual grouping has been employed successfully in several previous studies (see Driver et al., 2001; Kimchi \& RazpurkerApfeld, 2004; Russell \& Driver, 2005). Note, however, that our study separated the matrix from the distractor display spatially, rather than having them superimposed, further making it unlikely that the background distractors were attended (as in Kimchi \& Razpurker-Apfeld, 2004; Russell \& Driver, 2005). Taken together, our own adapted version of the paradigm, coupled with the fact that the participants suffered from neglect, strongly suggest that the patients neither overtly nor covertly attended to the contralesional background stimuli.

\section{Neglected Stimuli Can Influence Behavior}

The conclusion that neglected visual stimuli influence behavior is supported by the finding that the patients were influenced by task-irrelevant distractors presented in the neglected field. Distractor influence occurs to a similar extent in patients who have severe visuospatial deficits, as evidenced by their scores on the BIT and their very poor performance on the attentional-cuing task (i.e., PD) and in patients with milder impairment, as revealed by the BIT, and some residual ability to detect contralesional stimuli (i.e., GD) on the Posner covert attentional cuing task. This conclusion is further supported by the observation that the magnitude of the task-irrelevant distractor influence was similar across the groups for distractors presented across (i.e., in the neglected field for PDs and GDs) and within (i.e., within the intact right field) the same-hemifield. Furthermore, this finding is consistent with recent observations reporting that there is apparently normal activation in relatively early cortical visual areas, presumably in some of the same areas that subserve perceptual organization, in patients with neglect (Di Russo, Aprile, Spitoni, 
\& Spinelli, 2007; Rees et al., 2000; Vandenberghe et al., 2005; Vuilleumier et al., 2002; Vuilleumier et al., 2001).

Our findings are also compatible with several previous studies that have suggested that perceptually related — and even semantically related - unattended information from the neglected field may be processed to some extent in the absence of attention. For example, many studies of patients with neglect have shown processing of the contralesional stimulus when it can be grouped with the ipsilesional stimulus on the basis of bottom-up factors, such as color and proximity (Driver \& Halligan, 1991), or by brightness or collinear edges (Gilchrist, Humphreys, \& Riddoch, 1996; Rorden, Mattingley, Karnath, \& Driver, 1997). This is also true when the contralesional information is grouped with the right information by a global outline (Farah, Wallace, \& Vecera, 1993) and when the contralesional information forms the left side of an illusory contour (Kanizsa-type figure) of a partially occluded figure (Mattingley, Davis, \& Driver, 1997) or of any well-configured object or whole (Gilchrist et al., 1996; Humphreys \& Riddoch, 1994). It is also the case that task-irrelevant background information can affect performance implicitly by, for example, alleviating the midpoint deviation when patients with neglect perform line bisection (Esterman, McGlincheyBerroth, Alexander, \& Milberg, 2002; Ro \& Rafal, 1996). The influence of the unattended left-side information has also been observed in lexical tasks: Patients with neglect produced faster responses to a stimulus in the right field when it had been preceded by a brief presentation of an associated word in the neglected left field (Làdavas, Shallice, \& Zanella, 1997; Làdavas, Umiltà, \& Mapelli, 1997). This same patient was able neither to read the word in the left field aloud nor to judge the lexical or semantic content of the stimulus. Consistent with this, semantic priming from a contralesional picture to a foveally presented word has also been documented in neglect, suggesting that information on the left, albeit unattended and unavailable for explicit conscious report, is nevertheless processed to a substantial degree (McGlinchey-Berroth, Milberg, Verfaellie, Alexander, \& Kilduff, 1993). And, in a similar vein, physically different but related stimuli presented in the two hemifields of patients with neglect can be wellmatched despite the varying physical appearance, even though the stimulus on the left could not be recognized (Berti \& Rizzolatti, 1992).

Although the findings reported above are provocative and of great interest, many of these previous studies employed designs in which stimuli presented to the neglected field were associated with the stimuli presented on the right side of space by virtue of, for example, perceptual filling in, grouping by similarity, lexical relatedness, or semantic category, therefore inviting priming to occur not only from the left, neglected field to the right field, but perhaps vice versa, too (Boutsen \& Humphreys, 2000; Brooks, Wong, \& Robertson, 2005; Driver, Baylis, \& Rafal, 1992; Kumada \& Humphreys, 2001; Mattingley et al., 1997). Thus, making a lexical decision about a word presented foveally or on the ipsilesional right may be speeded by a semantically related word or picture on the left not only because the word or picture is processed a priori and able to influence the ipsilesional decision, but also perhaps because the robust, well-attended ipsilesional stimulus may prime the unattended contralesional stimulus in a backward fashion, and then, in turn, it supports the activation of the ipsilesional item (i.e., although the left is considered the prime stimulus and the right is considered the probe, these might operate in the opposite way). That such priming can occur even if the stimuli are not simultaneously present in the display as proactive and retroactive priming is possible; for examples, see empirical work by Behrmann, Moscovitch, Black, and Mozer (1990) and parallel computational studies by Mozer and Behrmann (1990), which illustrate some "pattern completion" of contralesional items by intact ipsilesional representations. A strength of the present study is that it eliminates this potential collaborative effort from the intact hemifield to the neglected hemifield. Indeed, there is no obvious way in which the well-attended ipsilesional matrix can affect the perception of the organizational structure of the contralesional background distractors; the information contained in each field is unrelated and inconsequential to the other. Also, because the samedifferent judgment on the ipsilesional matrix is so difficult, attention is well controlled to the intact side in this paradigm. The present study, therefore, goes beyond the existing studies and provides a compelling demonstration that task-irrelevant, ignored, and unrelated neglected information can still influence behavior.

\section{Distractor Filtering}

Our last and perhaps most counterintuitive conclusion is based on the finding that task-irrelevant distractors had a somewhat greater influence in patients than in controls in the cross-hemifield condition, but not in the same-hemifield condition. At first glance, this result might seem surprising: Why does neglected information influence behavior to a greater extent than unattended information does? This finding, however, is compatible with current theories of attentional control, in which attentional processing both enhances task-relevant information and suppresses task-irrelevant distractors (Friedman-Hill, Robertson, Desimone, \& Ungerleider, 2003; Humphreys et al., 2004; Moran \& Desimone, 1985; Motter, 1994; Reynolds, Chelazzi, \& Desimone, 1999). Thus, unattended information is subject to suppression, as in the control participants, but neglected information is not, and, consequently, task-irrelevant distractors exert greater influence in patients than in controls in the cross-hemifield condition, but not in the same-hemifield condition. This result, therefore, provides evidence for the dual roles of attentional selection - not only in enhancing target stimuli, but also in filtering out irrelevant distractors. A detailed analysis of the nature of the filtering of unattended information (or lack thereof) remains to be done, and elucidating the mechanisms of filtering and suppression in individuals with neglect offers a promising new avenue for understanding the representational dynamics of information processing in the unattended field. 


\section{Conclusion}

The present findings reveal that substantial perceptual processing - in this case, grouping by color similarity into rows/columns or into simple shapes - is achieved by the visual system without the necessary recruitment of attention. Thus, these results build on many previous studies and suggest that, even when attentional processing is disrupted, and even when it is not fully absent, perceptual organization can proceed apace.

\section{AUTHOR NOTE}

This work was supported by National Institute of Neurological Disorders and Stroke Grant No. NS07391-09 to S.S. and National Institute of Mental Health Grant No. MH54246 to M.B. We thank the patients and their families, as well as the participants of the Academy of Lifelong Learning at Carnegie Mellon University, for their participation in this research. Correspondence concerning this article should be addressed to S. Shomstein, Department of Psychology, George Washington University, Washington, DC 20052 (e-mail: shom@gwu.edu)

\section{REFERENCES}

Bahrami, B., Lavie, N., \& Rees, G. (2007). Attentional load modulates responses of human primary visual cortex to invisible stimuli. Current Biology, 17, 509-513.

Behrmann, M., Barton, J. J. S., Watt, S., \& Black, S. E. (1997). Impaired visual search in patients with unilateral neglect: An oculographic analysis. Neuropsychologia, 35, 1445-1458.

Behrmann, M., Moscovitch, M., Black, S. E., \& Mozer, M. (1990). Perceptual and conceptual factors underlying neglect dyslexia: Two contrasting case studies. Brain, 113, 1163-1183.

Berti, A., \& Rizzolatti, G. (1992). Is neglect a theoretically coherent unit? Neuropsychological Rehabilitation, 4, 111-114.

Biederman, I., Rabinowitz, J. C., Glass, A. L., \& Stacy, E. W., JR. (1974). On the information extracted from a glance at a scene. Journal of Experimental Psychology, 103, 597-600.

Boutsen, L., \& Humphreys, G. W. (2000). Axis-based grouping reduces visual extinction. Neuropsychologia, 38, 896-905.

Brooks, J. L., Wong, Y., \& RoberTson, L. C. (2005). Crossing the midline: Reducing attentional deficits via interhemispheric interactions. Neuropsychologia, $\mathbf{4 3}, 572-582$.

Cartwright-Finch, U., \& Lavie, N. (2007). The role of perceptual load in inattentional blindness. Cognition, 102, 321-340.

Chertkow, H., Bub, D. N., \& Seidenberg, M. (1989). Priming and semantic memory loss in Alzheimer's disease. Brain \& Language, 36, 420-446.

Corbetta, M., \& Shulman, G. L. (2002). Control of goal-directed and stimulus-driven attention in the brain. Nature Reviews Neuroscience, 3, 201-215.

Di Russo, F., Aprile, T., Spitoni, G., \& SPinelli, D. (2007). Impaired visual processing of contralesional stimuli in neglect patients: A visual-evoked potential study. Brain, 131, 842-854.

Driver, J., Baylis, G. C., \& Rafal, R. D. (1992). Preserved figureground segregation and symmetry perception in visual neglect. $\mathrm{Na}$ ture, 360, 73-75.

Driver, J., Davis, G., Russell, C., Turatto, M., \& Freeman, E. (2001). Segmentation, attention, and phenomenal visual objects. Cognition, 80, 61-95.

Driver, J., \& Halligan, P. W. (1991). Can visual neglect operate in object-centered co-ordinates? An affirmative single-case study. Cognitive Neuropsychology, 8, 475-496.

Enns, J. T., \& Kingstone, A. (1995). Access to global and local properties in visual search for compound stimuli. Psychological Science, 6, 283-291.

Esterman, M., McGlinchey-Berroth, R., Alexander, M. P., \& Milberg, W. (2002). Triangular backgrounds shift line bisection performance in hemispatial neglect: The critical point. Journal of the International Neuropsychological Society, 8, 721-726.

Farah, M. J., Wallace, M. A., \& Vecera, S. P. (1993). "What" and "where" in visual attention: Evidence from the neglect syndrome. In I. H. Robertson \& J. C. Marshall (Eds.), Unilateral neglect: Clinical and experimental studies (pp. 123-137). Hillsdale, NJ: Erlbaum.

Friedman-Hill, S. R., Robertson, L. C., Desimone, R., \& UngerLEIDER, L. G. (2003). Posterior parietal cortex and the filtering of distractors. Proceedings of the National Academy of Sciences, 100, 4263-4268.

Friedrich, F. J., Egly, R., Rafal, R. D., \& Beck, D. (1998). Spatial attention deficits in humans: A comparison of superior parietal and temporal-parietal junction lesions. Neuropsychology, 12, 193-207.

Gainotti, G., De Luca, L., Figliozzi, F., \& Doricchi, F. (2009). The influence of distracters, stimulus duration and hemianopia on first saccade in patients with unilateral neglect. Cortex, 45, 506-516.

Gilchrist, I. D., Humphreys, G. W., \& Riddoch, M. J. (1996). Grouping and extinction: Evidence for low-level modulation of visual selection. Cognitive Neuropsychology, 13, 1223-1256.

GriLl-SpeCtor, K., \& KANWISHER, N. (2005). Visual recognition: As soon as you know it is there, you know what it is. Psychological Science, 16, 152-160.

HiLLIS, A. E. (2006). Neurobiology of unilateral spatial neglect. Neuroscientist, 12, 153-163.

Humphreys, G. W., Kyllingsbek, S., Watson, D. G., Olivers, C. N. L., Law, I., \& Paulson, O. B. (2004). Parieto-occipital areas involved in efficient filtering in search: A time course analysis of visual marking using behavioural and functional imaging procedures. Quarterly Journal of Experimental Psychology, 57A, 610-635.

HumphreYs, G. W., \& RidDOCH, M. J. (1994). Attention to withinobject and between-object spatial representations: Multiple sites for visual selection. Cognitive Neuropsychology, 11, 207-241.

Karnath, H.-O., \& FetTer, M. (1995). Ocular space exploration in the dark and its relation to subjective and objective body orientation in neglect patients with parietal lesions. Neuropsychologia, 33, 371-377.

Kimchi, R., \& Peterson, M. A. (2008). Figure-ground segmentation can occur without attention. Psychological Science, 19, 660-668.

Kimchi, R., \& RAZPURKER-APFeLD, I. (2004). Perceptual grouping and attention: Not all groupings are equal. Psychonomic Bulletin \& Review, 11, 687-696.

Kumada, T., \& Humphreys, G. W. (2001). Lexical recovery from extinction: Interactions between visual form and stored knowledge modulate visual selection. Cognitive Neuropsychology, 18, 465-478.

Làdavas, E., Carletti, M., \& Gori, G. (1994). Automatic and voluntary orienting of attention in patients with visual neglect: Horizontal and vertical dimensions. Neuropsychologia, 32, 1195-1208.

LÀdavas, E., Petronio, A., \& Umiltà, C. (1990). The deployment of visual attention in the intact field of hemineglect patients. Cortex, 26, 307-317.

Làdavas, E., Shallice, T., \& Zanella, M. T. (1997). Preserved semantic access in neglect dyslexia. Neuropsychologia, 35, 257-270.

LÀdAVAS, E., Umiltà, C., \& MAPELli, D. (1997). Lexical and semantic processing in the absence of word reading: Evidence from neglect dyslexia. Neuropsychologia, 35, 1075-1085.

Làdavas, E., Umiltà, C., Ziani, P., Brogi, A., \& Minarini, M. (1993). The role of right side objects in left side neglect: A dissociation between perceptual and directional motor neglect. Neuropsychologia, 31, 761-773.

Lamy, D., Segal, H., \& Ruderman, L. (2006). Grouping does not require attention. Perception \& Psychophysics, 68, 17-31.

Lavie, N. (1995). Perceptual load as a necessary condition for selective attention. Journal of Experimental Psychology: Human Perception \& Performance, 21, 451-468.

LaVie, N., \& Robertson, I. H. (2001). The role of perceptual load in neglect: Rejection of ipsilesional distractors is facilitated with higher central load. Journal of Cognitive Neuroscience, 13, 867-876.

Lavie, N., \& Tsal, Y. (1994). Perceptual load as a major determinant of the locus of selection in visual attention. Perception \& Psychophysics, 56, 183-197.

Li, F. F., VanRullen, R., Koch, C., \& Perona, P. (2002). Rapid natural scene categorization in the near absence of attention. Proceedings of the National Academy of Sciences, 99, 9596-9601.

MACK, A., \& Rock, I. (1998). Inattentional blindness. Cambridge, MA: MIT Press.

Mack, A., Tang, B., Tuma, R., Kahn, S., \& Rock, I. (1992). Perceptual organization and attention. Cognitive Psychology, 24, 475-501. 
Mattingley, J. B., Davis, G., \& Driver, J. (1997). Preattentive fillingin of visual surfaces in parietal extinction. Science, 275, 671-674.

McGlinchey-Berroth, R., Milberg, W. P., Verfaellie, M., AlexANDER, M., \& Kilduff, P. T. (1993). Semantic processing in the neglected visual field: Evidence from a lexical decision task. Cognitive Neuropsychology, 10, 79-108.

Merikle, P. M., Smilek, D., \& Eastwood, J. D. (2001). Perception without awareness: Perspectives from cognitive psychology. Cognition, 79, 115-134.

Moore, C. M., \& Egeth, H. (1997). Perception without attention: Evidence of grouping under conditions of inattention. Journal of Experimental Psychology: Human Perception \& Performance, 23, 339-352.

Moran, J., \& Desimone, R. (1985). Selective attention gates visual processing in the extrastriate cortex. Science, 229, 782-784.

Motter, B. C. (1994). Neural correlates of feature selective memory and pop-out in extrastriate area V4. Journal of Neuroscience, 14, 2190-2199.

Mozer, M. C., \& Behrmann, M. (1990). On the interaction of selective attention and lexical knowledge: A connectionist account of neglect dyslexia. Journal of Cognitive Neuroscience, 2, 96-123.

Parton, A., Malhotra, P., \& Husain, M. (2004). Hemispatial neglect. Journal of Neurology, Neurosurgery \& Psychiatry, 75, 13-21.

PhILLIPS, W. A. (1974). On the distinction between sensory storage and short-term visual memory. Perception \& Psychophysics, 16, 283290

Posner, M. I., Snyder, C. R., \& Davidson, B. J. (1980). Attention and the detection of signals. Journal of Experimental Psychology: General, 109, 160-174.

Posner, M. I., Walker, J. A., Friedrich, F. J., \& Rafal, R. D. (1984). Effects of parietal injury on covert orienting of attention. Journal of Neuroscience, 4, 1863-1874.

Potter, M. C., \& Fox, L. F. (2009). Detecting and remembering simultaneous pictures in a rapid serial visual presentation. Journal of Experimental Psychology: Human Perception \& Performance, 35, 28-38.

Rees, G., Frith, C. D., \& Lavie, N. (1997). Modulating irrelevant motion perception by varying attentional load in an unrelated task. Science, 278, 1616-1619.

Rees, G., Wojciulik, E., Clarke, K., Husain, M., Frith, C., \& DrIVER, J. (2000). Unconscious activation of visual cortex in the damaged right hemisphere of a parietal patient with extinction. Brain, 123, 1624-1633.

Reynolds, J. H., Chelazzi, L., \& Desimone, R. (1999). Competitive mechanisms subserve attention in macaque areas V2 and V4. Journal of Neuroscience, 19, 1736-1753.

REYNolds, J. H., \& HeEger, D. J. (2009). The normalization model of attention. Neuron, 61, 168-185.

Ro, T., \& Rafal, R. D. (1996). Perception of geometric illusions in hemispatial neglect. Neuropsychologia, 34, 973-978.

Rorden, C., Mattingley, J. B., Karnath, H.-O., \& Driver, J. (1997). Visual extinction and prior entry: Impaired perception of temporal order with intact motion perception after unilateral parietal damage. Neuropsychologia, 35, 421-433.

Russell, C., \& Driver, J. (2005). New indirect measures of "inat- tentive" visual grouping in a change-detection task. Perception \& Psychophysics, 67, 606-623.

Shomstein, S., \& Yantis, S. (2006). Parietal cortex mediates voluntary control of spatial and nonspatial auditory attention. Journal of Neuroscience, 26, 435-439.

Torralba, A. (2003). Modeling global scene factors in attention. Journal of the Optical Society of America A, 20, 1407-1418.

Vandenberghe, R., Geeraerts, S., Molenberghs, P., Lafosse, C., Vandenbulcke, M., Peeters, K., et Al. (2005). Attentional responses to unattended stimuli in human parietal cortex. Brain, 128, 2843-2857.

Vuilleumier, P., Armony, J. L., Clarke, K., Husain, M., Driver, J., \& Dolan, R. J. (2002). Neural response to emotional faces with and without awareness: Event-related $\mathrm{CMRI}$ in a parietal patient with visual extinction and spatial neglect. Neuropsychologia, 40, 2156-2166.

Vuilleumier, P., Sagiv, N., Hazeltine, E., Poldrack, R. A., Swick, D., Rafal, R. D., \& Gabrieli, J. D. E. (2001). Neural fate of seen and unseen faces in visuospatial neglect: A combined eventrelated functional MRI and event-related potential study. Proceedings of the National Academy of Sciences, 98, 3495-3500.

WALKer, S., Stafford, P., \& Davis, G. (2008). Ultra-rapid categorization requires visual attention: Scenes with multiple foreground objects. Journal of Vision, 8(4, Art. 21), 1-12.

Wilson, B. A., Cockburn, J., \& Halligan, P. W. (1987). Behavioural Inattention Test. Bury St. Edmunds: Thames Valley Test Co.

Wolfe, J. M., Horowitz, T. S., \& Michod, K. O. (2007). Is visual attention required for robust picture memory? Vision Research, 47, 955-964.

Yantis, S., Schwarzbach, J., Serences, J. T., Carlson, R. L., SteinMetZ, M. A., Pekar, J. J., \& Courtney, S. M. (2002). Transient neural activity in human parietal cortex during spatial attention shifts. Nature Neuroscience, 5, 995-1002.

\section{NOTES}

1. We now resort to putting the word "unattended" in quotation marks to indicate the uncertainty of the extent to which the unattended stimuli are, in fact, unattended.

2. The checkerboard task was originally designed by Phillips (1974).

3 . The durations of the titrated displays were, on average, 367,635 , and $612 \mathrm{msec}$ for controls, good detectors, and poor detectors, respectively (see the text for group classifications).

4. It is important to note that the Posner cuing task is a measure of both exogenous and endogenous attention and, as such, is a powerful tool for identifying attentional deficits. The cue and a valid target serve as exogenous events; however, the cue that is followed by an invalid trial is a mixture of an exogenous orienting (following a cue) and an endogenous reorienting (when attention has to be redirected to the target position).

5 . This procedure is sometimes used for comparing priming magnitudes across groups with widely differing base RTs. For an example, see Chertkow, Bub, and Seidenberg (1989).

(Manuscript received March 25, 2009; revision accepted for publication October 14, 2009.) 